\title{
Interval Mapping of Quantitative Trait Loci for Time-to-Event Data with the Proportional Hazards Mixture Cure Model
}

\author{
Mengling Liu, ${ }^{1, *}$ Wenbin $\mathrm{Lu}^{2}{ }^{2}$ and Yongzhao Shao ${ }^{1}$ \\ ${ }^{1}$ Division of Biostatistics, School of Medicine, New York University, 650 First Avenue, \\ 5th Floor, New York, New York 10016, U.S.A. \\ ${ }^{2}$ Department of Statistics, North Carolina State University, 2501 Founders Drive, Raleigh, \\ North Carolina 27695, U.S.A. \\ *email: mengling.liu@med.nyu.edu
}

\begin{abstract}
SummaRY. Interval mapping using normal mixture models has been an important tool for analyzing quantitative traits in experimental organisms. When the primary phenotype is time-to-event, it is natural to use survival models such as Cox's proportional hazards model instead of normal mixtures to model the phenotype distribution. An extra challenge for modeling time-to-event data is that the underlying population may consist of susceptible and nonsusceptible subjects. In this article, we propose a semiparametric proportional hazards mixture cure model which allows missing covariates. We discuss applications to quantitative trait loci (QTL) mapping when the primary trait is time-to-event from a population of mixed susceptibility. This model can be used to characterize QTL effects on both susceptibility and time-to-event distribution, and to estimate QTL location. The model can naturally incorporate covariate effects of other risk factors. Maximum likelihood estimates for the parameters in the model as well as their corresponding variance estimates can be obtained numerically using an EM-type algorithm. The proposed methods are assessed by simulations under practical settings and illustrated using a real data set containing survival times of mice after infection with Listeria monocytogenes. An extension to multiple intervals is also discussed.
\end{abstract}

KeY words: Age-at-onset; Cure model; EM algorithm; Proportional hazards model; QTL mapping.

\section{Introduction}

With the development of genetic markers and genetic linkage maps, it is now possible to detect and localize chromosomal regions having significant effects on a quantitative trait. These regions are often called quantitative trait loci (QTL). Identification and characterization of QTL have major economic importance to animal and plant breeders as well as potential medical importance for treating complex human diseases (Lander and Schork, 1994). In practice, QTL analysis is carried out in multiple stages. For example, Shalom and Darvasi (2002) summarized these stages into four parts: QTL detection, QTL mapping, fine mapping, and gene cloning. The first stage tests the hypothesis of linkage between the putative QTL and markers and is usually accomplished by a series of likelihood ratio tests (LRT) across a chromosome(s). The second stage estimates the QTL location and effects and may be carried out by maximum likelihood estimates (MLE). Although different researchers might classify those stages differently, all seem to agree that both detection of QTL and estimation of location and effects are important and each stage may require different statistical procedures (Darvasi et al., 1993).

In standard interval mapping of the QTL, the phenotype distribution is modeled as a mixture of two (or more) components corresponding to two (or more) different genotypes at the putative QTL (Lander and Botstein, 1989).
The distributions of the mixture components are typically assumed to be normal with the same variance but different means. For some time-to-event phenotypes, such as age-atonset, some simple transformation is often used to achieve approximate normality. However, the choice of transformation is somewhat arbitrary and hard to justify. Nonparametric methods have been developed to test the presence of QTL (Kruglyak and Lander, 1995; Broman, 2003); however, these methods do not provide consistent estimation for the QTL effects.

In some genetic studies with survival end points, the population under study consists of susceptible and nonsusceptible individuals. All susceptible subjects would eventually experience the event in the absence of censoring, while nonsusceptible subjects are not at risk of developing such events and can be regarded as "cured." For example, in a data set consisting of 116 female intercross mice after infection with Listeria monocytogenes (Boyartchuk et al., 2001; Broman, 2003), a substantial proportion of the mice survived after 240 hours and may be considered as cured. Kaplan-Meier curves for survival times of three groups with different genotypes at the marker D13M147 on chromosome 13 are plotted in Figure 1a. Notably, the two survival curves corresponding to genotypes $A a$ and $a a$ level off nearly above 0.4 at the right tail, while the survival curve of the mice with genotype $A A$ levels off around 0.1 at the right tail. In addition, mice with genotype 


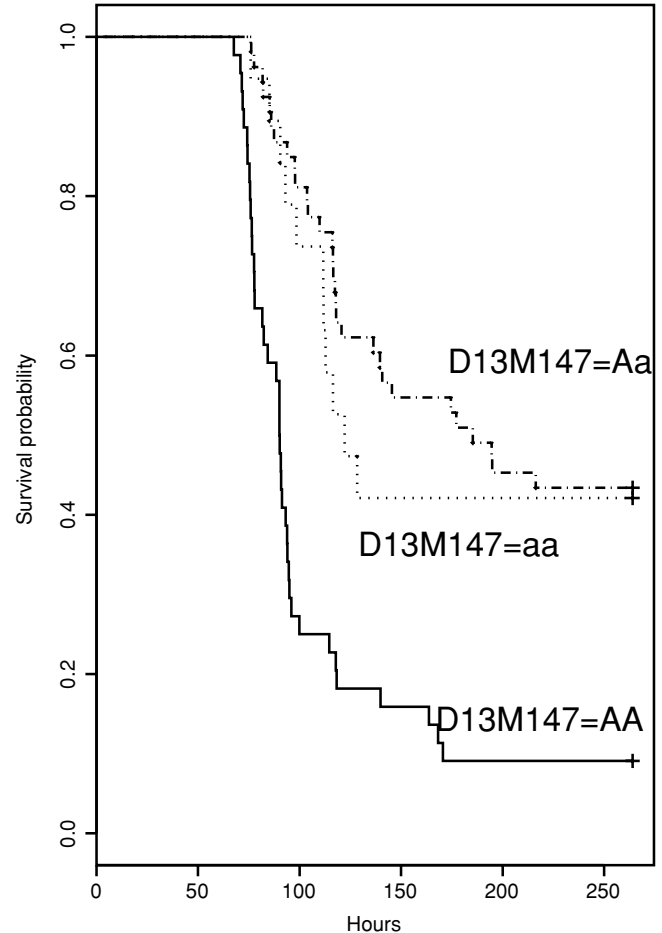

(a)

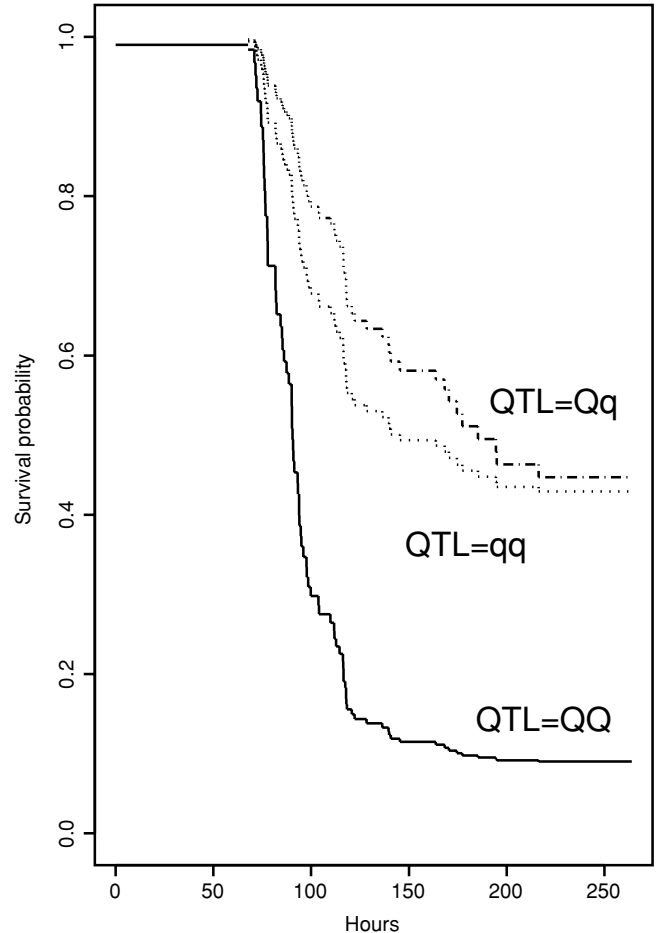

(b)

Figure 1. Survival distributions. (a) Kaplan-Meier survival curves based on the observed data. (b) Estimated survival curves based on proportional hazards mixture cure model.

$A a$ tend to have more late events than those with genotypes $A A$ and $a a$. This indicates that both the distribution of event times and the cure proportion may vary among the different genotypic groups of mice. Broman (2003) proposed a twopart model: a normal distribution for log survival times of susceptible subjects and a point mass at the end of study for nonsusceptible subjects. Mice still alive at the end of the study are all assumed to be nonsusceptible (cured). This simple way of identifying cured individuals may be appropriate for these particular data; however, it is not applicable to the common survival data with random right censoring. Feenstra and Skovgaard (2004) also studied a similar problem and suggested using a two-component mixture model by introducing a latent component indicator; however, both the normality assumption of the components and equality of their variances appear questionable.

Recently, semiparametric cure models have received much attention in the analysis of survival data with long-term survivors (Kuk and Chen, 1992; Peng and Dear, 2000; Sy and Taylor, 2000; Lu and Ying, 2004, among others). One such model is the so-called proportional hazards cure model in which the proportional hazards regression (Cox, 1972) is used to model time-to-event of susceptible subjects, while logistic regression is used to model the cure probability. The semiparametric proportional hazards cure model provides a useful tool in QTL analysis when the primary phenotype is time-to-event in the presence of nonsusceptible subjects. The challenges to be addressed for this approach include dealing with the missing genotype information at the putative QTL and the partially missing susceptibility status due to random censoring.
Our research, as well as Broman (2003), among others, have considered the important and challenging problem of detecting the existence of QTL for time-to-event traits from a population of mixed susceptibility. In this article, we focus on joint estimation of QTL location and effects using the proportional hazards mixture cure model and on developing the corresponding statistical inference procedures. The rest of the article is organized as follows. Section 2 introduces notation and the proposed model. In Section 3, a modified expectation-maximization (EM) algorithm is proposed to obtain maximum likelihood estimates for the parameters. Two different methods to estimate standard errors are also investigated. Section 4 is devoted to simulation studies and the analysis of Listeria monocytogenes data. In some situations, the uncertainty of target region may extend over multiple marker intervals, and thus an extended estimation approach is developed and demonstrated with supplementary simulation study in Section 5. Some concluding remarks are given in Section 6.

\section{Notation and Model Specifications}

Our discussion will focus on inbreeding experimental crosses, such as backcross and intercross $\left(F_{2}\right)$ designs. Such cross designs provide efficient ways to study variations in the quantitative trait caused by possible genetic factors (Lander and Schork, 1994; Doerge, Zeng, and Weir, 1997). Consider $n$ individuals in the study. Let $T_{i}$ denote the potential time-to-event for the $i$ th subject. Then $T_{i}$ has the following decomposition:

$$
T_{i}=\eta_{i} T_{i}^{*}+\left(1-\eta_{i}\right) \infty
$$


where $T_{i}^{*}<\infty$ denotes the failure time if the subject is susceptible and $\eta_{i}$ indicates, by the value 1 or 0 , whether the $i$ th subject is susceptible or not. The observed event time is $Y_{i}$ $=\min \left(T_{i}, C_{i}\right)$, where $C_{i}$ is the random censoring time and is assumed to be noninformative (Kalbfleisch and Prentice, 2002). The censoring indicator is denoted by $\delta_{i}=I\left(T_{i} \leq C_{i}\right)$. It is worth pointing out that $\delta_{i}=1$ implies $\eta_{i}=1$ and otherwise, $\eta_{i}$ is unobservable when $\delta_{i}=0$. Thus the susceptible indicators $\left\{\eta_{i}\right\}$ can only be partially observed due to censoring. Let $G_{i}$ denote the genotypic information of the putative QTL and $M_{i}$ denote the genotypes of two flanking markers of a target interval. In the backcross design, $G_{i}=1 / 0$ stands for the genotypes $Q Q / Q q$, respectively, and $M_{i}$ takes $2^{2}$ possible combinations; in the intercross design, $G_{i}$ can be coded using a two-dimensional vector with three possible values, $(1,0)$, $(0,1)$, and $(0,0)$, according to the genotypes $Q Q, Q q$, and $q q$, respectively, and $M_{i}$ has $3^{2}$ possible combinations. In addition, let $Z_{i}$ denote other covariates of interest, such as environmental factors, which are assumed to be independent of $G_{i}$. The complete data would consist of $\left\{T_{i}^{*}, C_{i}, \delta_{i}, \eta_{i}, G_{i}, M_{i}, Z_{i}\right.$; $i=1, \ldots, n\}$, while the observed data consist of independent copies of $\left\{Y_{i}, \delta_{i}, M_{i}, Z_{i}\right\}$.

A logistic regression model is assumed for the susceptibility indicator $\eta_{i}$

$$
\pi_{i}\left(G_{i}\right) \equiv \pi\left(G_{i}, Z_{i}^{*}\right) \equiv \operatorname{Pr}\left(\eta_{i}=1 \mid G_{i}, Z_{i}^{*}\right)=\frac{e^{\gamma^{\prime} Z_{i}^{*}+\gamma_{g}^{\prime} G_{i}}}{1+e^{\gamma^{\prime} Z_{i}^{*}+\gamma_{g}^{\prime} G_{i}}},
$$

where $Z_{i}^{*}=\left(1, Z_{i}^{\prime}\right)^{\prime}$ so that $\gamma$ contains the intercept term. The Cox proportional hazards model is used for the failure time $T^{*}$ of a susceptible subject and the hazard function is defined as

$$
\lambda\left(t \mid G_{i}, Z_{i}\right)=\lambda_{0}(t) e^{\beta^{\prime} Z_{i}+\beta_{g}^{\prime} G_{i}},
$$

where $\lambda_{0}(t)$ is an unspecified baseline hazard function, and $\Lambda_{0}(t)=\int_{0}^{t} \lambda_{0}(s) d s$ denotes the cumulative hazard function. If the genotypes $\left\{G_{i}\right\}$ of the QTL are known, models (2) and (3) specify the conventional proportional hazards cure model for which the inferential methods have been investigated by a number of authors (Kuk and Chen, 1992; Peng and Dear, 2000; Sy and Taylor, 2000; Lu and Ying, 2004, among others). A difficulty of applying the model specified by (2) and (3) directly is that genotype $\left\{G_{i}\right\}$ of the QTL are not observed, only the genotypes of flanking markers $\left\{M_{i}\right\}$ are available. Suppose that the QTL is flanked by markers $M_{L}$ and $M_{U}$. The recombination fraction $\theta$ between these two markers is known because the distance between $M_{L}$ and $M_{R}$ is observed. Let $r$ and $s$ denote the recombination fractions between the QTL and $M_{L}$ and between the QTL and $M_{U}$, respectively. Under the assumption of no interference, it is known that $\theta=r+s-2 r s$, and hence $s=(\theta-r) /(1-2 r)$. When $\theta$ is relatively small, $r+s$ approximates $\theta$. The conditional probability $p_{i}=\operatorname{Pr}\left(G_{i}=1 \mid M_{i}\right)$ is a function of $\theta, r$, and $s$, and its formulation is available in many genetics papers and books, for example, Lynch and Walsh (1998, p. 435).

The parameters of interest in the model consist of $\boldsymbol{\alpha}=$ $\left(\gamma, \gamma_{g}, \beta, \beta_{g}, r\right)$, where $\left(\gamma, \gamma_{g}\right)$ represent the long-term effects of QTL and environmental covariates on susceptibility, and $\left(\beta, \beta_{g}\right)$ represent the short-term effects on time-to-event for susceptible subjects. A modified EM algorithm is proposed in the next section for simultaneous estimation of both the parameter vector $\boldsymbol{\alpha}$ and the infinite-dimensional parameter $\Lambda_{0}(t)$.

\section{Statistical Inference Procedures \\ 3.1 A Modified EM Algorithm}

The likelihood given the complete data $\left(Y_{i}, \delta_{i}, \eta_{i}, G_{i}, M_{i}, Z_{i}\right)$ is

$$
\begin{aligned}
L_{C}\left(\boldsymbol{\alpha}, \Lambda_{0}\right)= & \prod_{i=1}^{n}\left(\frac{e^{\gamma^{\prime} Z_{i}^{*}+\gamma_{g}^{\prime} G_{i}}}{1+e^{\gamma^{\prime} Z_{i}^{*}+\gamma_{g}^{\prime} G_{i}}}\right)^{\eta_{i}}\left(\frac{1}{1+e^{\gamma^{\prime} Z_{i}^{*}+\gamma_{g}^{\prime} G_{i}}}\right)^{1-\eta_{i}} \\
& \times \prod_{i=1}^{n}\left\{\lambda_{0}\left(Y_{i}\right) e^{\beta^{\prime} Z_{i}+\beta_{g}^{\prime} G_{i}}\right\}^{\delta_{i} \eta_{i}} e^{-\eta_{i} \Lambda_{0}\left(Y_{i}\right) \exp \left(\beta^{\prime} Z_{i}+\beta_{g}^{\prime} G_{i}\right)} \\
& \times \prod_{i=1}^{n} \operatorname{pr}\left(G_{i} \mid M_{i}\right) \\
= & L_{1}\left(\gamma, \gamma_{g}\right) \cdot L_{2}\left(\beta, \beta_{g}, \Lambda_{0}\right) \cdot L_{3}(r)
\end{aligned}
$$

This complete-data likelihood $L_{C}$ consists of three parts: the logistic model, the Cox proportional hazards model, and the conditional probability of $G_{i}$ given the flanking markers' genotypes $M_{i}$. Such a factorization of the complete-data likelihood is favorable for using the EM algorithm (Dempster, Laird, and Rubin, 1977). We will use $L$ to denote a likelihood function and $l$ the corresponding log likelihood.

In the E-step, we will calculate the conditional expectation of $l_{C}\left(\boldsymbol{\alpha}, \Lambda_{0}\right)$ with respect to the unobserved quantities $\left\{\eta_{i}, G_{i}\right\}$ given the current estimated parameter values and the observed data $\mathcal{O}_{i}=\left\{Y_{i}, \delta_{i}, M_{i}, Z_{i}\right\}$. Here we only present the formula under backcross design for illustration and similar formulas can also be derived for intercross design. Let $P_{i}^{(k)}=\left\{P_{\eta_{i}}^{(k)}, P_{G_{i}}^{(k)}, P_{\eta G_{i}}^{(k)}\right\}$ denote the corresponding conditional expectations of $\left\{\eta_{i}, G_{i}, \eta_{i} G_{i}\right\}$ given the observed data and the $k$ th step parameter estimates $\left(\boldsymbol{\alpha}^{(k)}, \Lambda_{0}^{(k)}\right)$. The conditional expectation of the log complete-data likelihood is denoted by $\tilde{l}_{C}\left(\boldsymbol{\alpha}, \Lambda_{0} \mid \boldsymbol{\alpha}^{(k)}, \Lambda_{0}^{(k)}\right)$ and its detailed expression is given in the Appendix. It is evident that the computation of $\tilde{l}_{C}$ only involves $P_{i}^{(k)} \equiv\left\{P_{\eta_{i}}^{(k)}, P_{G_{i}}^{(k)}, P_{\eta G_{i}}^{(k)}\right\}, i=1, \ldots, n$. These quantities can be calculated as

$$
\begin{aligned}
& P_{\eta_{i}}^{(k)}=E\left(\eta_{i} \mid \mathcal{O}_{i}, \boldsymbol{\alpha}^{(k)}, \Lambda_{0}^{(k)}\right) \\
& = \begin{cases}1 & \delta_{i}=1 \\
\frac{e^{-\Lambda_{0}\left(Y_{i}\right) \exp \left(\beta^{\prime} Z_{i}+\beta_{g}\right)} \pi_{i}(1) p_{i}+e^{-\Lambda_{0}\left(Y_{i}\right) \exp \left(\beta^{\prime} Z_{i}\right)} \pi_{i}(0)\left(1-p_{i}\right)}{D_{i 0}} & \delta_{i}=0,\end{cases} \\
& P_{G_{i}}^{(k)}=E\left(G_{i} \mid \mathcal{O}_{i}, \boldsymbol{\alpha}^{(k)}, \Lambda_{0}^{(k)}\right) \\
& = \begin{cases}\frac{e^{\beta_{g}} e^{-\Lambda_{0}\left(Y_{i}\right) \exp \left(\beta^{\prime} Z_{i}+\beta_{g}\right)} \pi_{i}(1) p_{i}}{D_{i 1}} & \delta_{i}=1 \\
\frac{e^{-\Lambda_{0}\left(Y_{i}\right) \exp \left(\beta^{\prime} Z_{i}+\beta_{g}\right)} \pi_{i}(1) p_{i}+\left\{1-\pi_{i}(1)\right\} p_{i}}{D_{i 0}} & \delta_{i}=0,\end{cases} \\
& P_{\eta G_{i}}^{(k)}=E\left(\eta_{i} G_{i} \mid \mathcal{O}_{i}, \boldsymbol{\alpha}^{(k)}, \Lambda_{0}^{(k)}\right) \\
& = \begin{cases}\frac{e^{\beta_{g}} e^{-\Lambda_{0}\left(Y_{i}\right) \exp \left(\beta^{\prime} Z_{i}+\beta_{g}\right)} \pi_{i}(1) p_{i}}{D_{i 1}} & \delta_{i}=1 \\
\frac{e^{-\Lambda_{0}\left(Y_{i}\right) \exp \left(\beta^{\prime} Z_{i}+\beta_{g}\right)} \pi_{i}(1) p_{i}}{D_{i 0}} & \delta_{i}=0,\end{cases}
\end{aligned}
$$


where $\pi_{i}$ is given in equation (2), and

$$
\begin{aligned}
D_{i 1}= & e^{\beta_{g}} e^{-\Lambda_{0}\left(Y_{i}\right) \exp \left(\beta^{\prime} Z_{i}+\beta_{g}\right)} \pi_{i}(1) p_{i} \\
& +e^{-\Lambda_{0}\left(Y_{i}\right) \exp \left(\beta^{\prime} Z_{i}\right)} \pi_{i}(0)\left(1-p_{i}\right), \\
D_{i 0}= & \left\{e^{-\Lambda_{0}\left(Y_{i}\right) \exp \left(\beta^{\prime} Z_{i}+\beta_{g}\right)} \pi_{i}(1)+1-\pi_{i}(1)\right\} p_{i} \\
& +\left\{e^{-\Lambda_{0}\left(Y_{i}\right) \exp \left(\beta^{\prime} Z_{i}\right)} \pi_{i}(0)+1-\pi_{i}(0)\right\}\left(1-p_{i}\right) .
\end{aligned}
$$

Note that to simplify the notation, the superscript $(k)$ of parameters $\boldsymbol{\alpha}$ and $\Lambda_{0}$ has been suppressed in the formulas (righthand side) above.

From equation (4) and the Appendix, the conditional expectation of the log complete-data likelihood can be expressed as $\tilde{l}_{C} \equiv \tilde{l}_{1}\left(\gamma, \gamma_{g} ; P^{(k)}, \mathcal{O}\right)+\tilde{l}_{2}\left(\beta, \beta_{g}, \Lambda_{0}(t) ; P^{(k)}, \mathcal{O}\right)+$ $\tilde{l}_{3}\left(r ; P^{(k)}, \mathcal{O}\right)$, where $\tilde{l}\left(\cdot ; P^{(k)}, \mathcal{O}\right)$ denotes conditional expectation of corresponding log likelihood given observed data and current parameter estimates, $P^{(k)}=\left\{P_{i}^{(k)}: i=1, \ldots, n\right\}$ and $\mathcal{O}=\left\{\mathcal{O}_{i}: i=1, \ldots, n\right\}$. It is clear that each component of $\tilde{l}_{C}$ only involves its own subset of the parameters and thus the maximization of $\tilde{l}_{C}$ in the M-step can be conducted separately for each component. Since $\tilde{l}_{1}$ and $\tilde{l}_{3}$ only involve finite-dimensional parameters, the maximization can be easily achieved. To maximize $\tilde{l}_{2}$ over $\left(\beta, \beta_{g}\right)$ and $\Lambda_{0}(t)$, we implement the profile likelihood technique, which first computes the nonparametric MLE of $\Lambda_{0}(t)$ for each fixed $\left(\beta, \beta_{g}\right)$. A nonparametric MLE of $\Lambda_{0}(t)$ at the $k$ th step is given by the Nelson-Aalen-type estimator (Breslow, 1975; Kalbfleisch and Prentice, 2002):

$$
\begin{aligned}
& \Lambda_{0}^{(k+1)}\left(t ; \beta, \beta_{g}\right) \\
& =\sum_{i: Y_{i} \leq t}\left[\frac{\delta_{i}}{\sum_{j \in R_{i}}\left\{e^{\beta^{\prime} Z_{j}+\beta_{g}} P_{\eta G_{j}}^{(k)}+e^{\beta^{\prime} Z_{j}}\left(P_{\eta_{j}}^{(k)}-P_{\eta G_{j}}^{(k)}\right)\right\}}\right], \\
& 0<t<\infty,
\end{aligned}
$$

where $R_{i}$ is the at-risk set at $Y_{i}-$. Plugging $\Lambda_{0}^{(k+1)}(t)$ into $\tilde{l}_{2}$ yields the profile log likelihood $\tilde{l}_{2}^{p}=\tilde{l}_{2}^{p}\left(\beta, \beta_{g} ; P^{(k)}, \mathcal{O}\right)$ for $\left(\beta, \beta_{g}\right)$, that is,

$$
\begin{aligned}
\tilde{l}_{2}^{p}=\sum_{i=1}^{n} \delta_{i} & {\left[\beta^{\prime} Z_{i}+\beta_{g}^{\prime} P_{G_{i}}^{(k)}\right.} \\
& \left.-\log \sum_{j \in R_{i}}\left\{e^{\beta^{\prime} Z_{j}+\beta_{g}} P_{\eta G_{j}}^{(k)}+e^{\beta^{\prime} Z_{j}}\left(P_{\eta_{j}}^{(k)}-P_{\eta G_{j}}^{(k)}\right)\right\}\right] .
\end{aligned}
$$

Then $\tilde{l}_{2}^{p}$ is maximized to obtain updated estimators $\left(\beta^{(k+1)}\right.$, $\left.\beta_{g}^{(k+1)}\right)$ for $\left(\beta, \beta_{g}\right)$. The updated estimator for $\Lambda_{0}$ is just $\Lambda_{0}^{(k+1)}(t)=\Lambda_{0}^{(k+1)}\left(t ; \beta^{(k+1)}, \beta_{g}^{(k+1)}\right)$. The steps of the proposed EM algorithm at the $k$ th iteration are summarized as follows:

(1) Given $\boldsymbol{\alpha}^{(k)}$ and $\Lambda_{0}^{(k)}$, calculate the conditional expectations $P^{(k)}$.

(2) Update $\left(\gamma, \gamma_{g}\right)$ by maximizing the expected log likelihood $\tilde{l}_{1}\left(\gamma, \gamma_{g} ; P^{(k)}, \mathcal{O}\right)$.

(3) Update $\left(\beta, \beta_{g}\right)$ by maximizing the expected log profile likelihood $\tilde{l}_{2}^{p}\left(\beta, \beta_{g} ; P^{(k)}, \mathcal{O}\right)$.

(4) Update $\Lambda_{0}$ by plugging the estimators of $\left(\beta, \beta_{g}\right)$ obtained in step 3 into (5).
(5) Update $r$ by maximizing the expected log likelihood $\tilde{l}_{3}\left(r ; P^{(k)}, \mathcal{O}\right)$.

The above steps $1-5$ will be iterated until a predetermined convergence criterion is met. Convergence of the objective likelihood function $\left(\tilde{l}_{C}\right)$ is used as the criterion for the EM algorithm both in our simulation studies and in the analysis of real data. Specifically, we consider the convergence criterion of $\left|\tilde{l}_{C}^{k+1}-\tilde{l}_{C}^{k}\right|<\varepsilon$ and choose $\varepsilon=0.01$ in our numerical studies.

\subsection{Variance Estimation and Statistical Inference}

Under the context considered here, variance estimation is complicated by multiple factors including the unknown genotypes $G_{i}$ 's, the partially missing information of cure status indicators $\eta_{i}$ 's, and the presence of the infinite-dimensional parameter $\Lambda_{0}(t)$. If the infinite-dimensional parameter $\Lambda_{0}(t)$ is known up to a parametric form, the estimation procedure becomes an ordinary parametric EM algorithm. Then the variance-covariance formula proposed by Louis (1982) provides valid variance estimates. On the other hand, if $\left(G_{i}, \eta_{i}\right)$ are known, the model involves no missing information and becomes an ordinary semiparametric model. For such semiparametric models, Johansen (1983) and Murphy and Van der Vaart (2000) showed that the inverse of the information matrix of the profile likelihood can produce valid variance estimates for the finite-dimensional parameters of interest. A number of methods have been proposed in the literature for variance estimation in semiparametric models with missing covariates (Klein, 1992; Li and Thompson, 1997; Chen and Little, 1999). Here we explore two commonly used approaches, and investigate the performance of these two methods under practical settings in the next section using simulations.

Method 1: Louis's formula based on complete-data profile likelihood: To combine the strength of Louis's (1982) formula for handling missing data and the profile likelihood for dealing with high-dimensional nuisance parameters, we apply Louis's formula to the complete-data profile likelihood, $L_{C}^{p l}=L_{1}(\gamma$, $\left.\gamma_{g}\right) \cdot P L_{2}\left(\beta, \beta_{g}\right) \cdot L_{3}(r)$, where $P L_{2}\left(\beta, \beta_{g}\right)$ is obtained by profiling out the infinite-dimensional parameter $\Lambda_{0}(t)$ from $L_{2}\left(\beta, \beta_{g}, \Lambda_{0}\right)$ (Kalbfleisch and Prentice, 2002) and is given by

$$
P L_{2}\left(\beta, \beta_{g}\right)=\prod_{i=1}^{n}\left\{\frac{\exp \left(\beta^{\prime} Z_{i}+\beta_{g}^{\prime} G_{i}\right)}{\sum_{j \in R_{i}} \eta_{j} \exp \left(\beta^{\prime} Z_{j}+\beta_{g}^{\prime} G_{j}\right)}\right\}^{\delta_{i}} .
$$

The $P L_{2}$ in (7) can also be viewed as a modification of the partial likelihood for the Cox proportional hazards model in order to take into consideration the latent susceptibility. The combination of Louis's formula and profile likelihood was also used in Li and Thompson (1997) to estimate variance of parameter estimates in a proportional hazards frailty model with major gene effects for family data. It can avoid inversion of the high-dimensional information matrix for semiparametric models. Note that in Louis's formula (Louis, 1982) for the ordinary parametric EM algorithm, the third term is virtually zero. However, for the semiparametric model we considered, keeping the third term is helpful for improving the accuracy. Meanwhile, the formula indicates clearly that the derivative 
with respect to different subsets of parameters only depends on its own part of the likelihood (or profile likelihood).

Method 2: EM-aided numerical differentiation: The second method computes the observed information matrix using the so-called EM-aided differentiation method, which was first studied by Meilijson (1989) for parametric models. It has been extended to the proportional hazards model with missing covariates by Chen and Little (1999) who also derived its theoretical justification. To estimate the covariance matrix of parameter estimates, this method computes the numerical differentiation of the observed profile likelihood score functions, which is the same as the conditional expectation of the complete-data log-likelihood score at the convergent point. For example, consider estimating the variance of the MLE for $\beta_{*} \equiv\left(\beta, \beta_{g}\right)$. Let $\boldsymbol{\alpha}\left(\beta_{*}\right)=\left(\gamma\left(\beta_{*}\right), \gamma_{g}\left(\beta_{*}\right), r\left(\beta_{*}\right), \Lambda_{0}\left(\beta_{*}\right)\right)$ denote the maximizer of the observed likelihood with $\beta_{*}$ fixed. Following Chen and Little (1999), the information matrix for $\beta_{*}$ can be calculated as follows:

(1) Fix $\beta_{*}$ at its MLE $\hat{\beta}_{*}$, and run the EM algorithm to obtain $\boldsymbol{\alpha}\left(\hat{\beta}_{*}\right)$.

(2) Perturb the $j$ th component of $\beta_{*}$ by a small amount $h$ in the neighborhood of $\hat{\beta}_{*}$, denoted by $\hat{\beta}_{* j}$, and run the EM algorithm to obtain $\boldsymbol{\alpha}\left(\hat{\beta}_{* j}\right)$.

(3) Approximate the $i$ th row of the information matrix of $\beta_{*}$ by

$$
\begin{aligned}
\frac{1}{h}\left[E\left\{l_{2}^{\beta_{*}}\left(\hat{\beta}_{* j}, \Lambda_{0}\left(\hat{\beta}_{* j}\right)\right) \mid \hat{\beta}_{* j}, \boldsymbol{\alpha}\left(\hat{\beta}_{* j}\right), \mathcal{O}\right\}\right. \\
\left.-E\left\{l_{2}^{\beta_{*}}\left(\hat{\beta}_{*}, \Lambda_{0}\left(\hat{\beta}_{*}\right)\right) \mid \hat{\beta}_{*}, \boldsymbol{\alpha}\left(\hat{\beta}_{*}\right), \mathcal{O}\right\}\right],
\end{aligned}
$$

where $l_{2}^{\beta^{*}}\left(\hat{\beta}_{*}, \Lambda_{0}\left(\hat{\beta}_{*}\right)\right)=\left.\frac{\partial l_{2}\left(\beta^{*}, \Lambda_{0}\right)}{\partial \beta_{*}}\right|_{\beta_{*}=\hat{\beta}_{*}, \Lambda_{0}=\Lambda_{0}\left(\hat{\beta}_{*}\right)}$ denotes the derivative of complete-data log likelihood with respect to $\beta_{*}$ evaluated at $\left(\hat{\beta}_{*}, \Lambda_{0}\left(\hat{\beta}_{*}\right)\right)$.

Chen and Little (1999) also recommended to perturb the parameters in both directions and suggested the perturbation size $h=1 / n$, where $n$ is the sample size.

The advantages of Method 1 include its ease in understanding and implementation. Method 2 numerically calculates the second derivative of the observed log profile likelihood with respect to the parameters of interest; therefore, it does not require derivation of the second derivatives of the log likelihood analytically. The corresponding theoretical justification can be similarly derived as that in Chen and Little (1999).

The variance estimates can be obtained by either method each with its own advantages. A Wald-type 95\% confidence interval for $\boldsymbol{\alpha}$ can be constructed as $\hat{\boldsymbol{\alpha}} \pm 1.96 \cdot \hat{\mathrm{SE}}(\hat{\boldsymbol{\alpha}})$. But for the recombination fraction parameter $r$, which is restricted in $[0, \theta]$, such a confidence interval may produce unreasonable values falling outside the range $[0, \theta]$. So we propose to reparameterize $r$ using $r^{*}=\operatorname{logit}(r / \theta)$, for which the range is $(-\infty$, $\infty)$. Then the confidence interval for $r$ can be constructed as $\left[\theta \frac{e^{r_{L}^{*}}}{1+e^{r_{L}^{*}}}, \theta \frac{e^{r_{U}^{*}}}{1+e_{U}^{r^{*}}}\right]$, where $r_{L}^{*}$ and $r_{U}^{*}$ are the lower and upper endpoints, respectively, of the confidence interval for $r^{*}$. This reparameterization can also reduce the computational complexity because it simplifies a constrained maximization problem to a maximization problem over the whole real line.

\section{Numerical Results}

\subsection{Simulation Study}

To assess the performance of the proposed method under practical settings, simulations were conducted using a backcross design with moderate sample size. We consider the following proportional hazards cure model,

$$
\begin{gathered}
\log \left\{\frac{\operatorname{Pr}\left(\eta_{i}=1\right)}{1-\operatorname{Pr}\left(\eta_{i}=1\right)}\right\}=\gamma_{0}+\gamma_{1} Z_{i}+\gamma_{g} G_{i}, \quad \text { and } \\
\lambda\left(t \mid Z_{i}, G_{i}, \eta_{i}=1\right)=\lambda_{0}(t) e^{\beta_{1} Z_{i}+\beta_{g} G_{i}},
\end{gathered}
$$

where $G_{i}=1$ if the genotype of the QTL is $Q Q$ and $G_{i}=0$ for $Q q$. The covariate $Z_{i} \sim \operatorname{Bernoulli}(0.5)$ is a binary covariate that mimics an exposure indicator, for example, smoking indicator. We set $\gamma_{0}=1, \gamma_{1}=0.5, \gamma_{g}=-2, \lambda_{0}(t)=1, \beta_{1}=0.5$, and $\beta_{g}=-1$ in the above model, under which the cure fraction is $37.8 \%$ for exposed $\left(Z_{i}=1\right)$ carriers $(Q Q)$ and $73.1 \%$ for nonexposed $\left(Z_{i}=0\right)$ noncarriers $(Q q)$. This also gives the average survival time of 1.65 for susceptible exposed carriers and 1 for susceptible nonexposed noncarriers. The setting is similar to the real data set in the sense that the group with higher cure rate can have shorter survival time once they are susceptible. The independent censoring times $\left(C_{i}\right)$ were generated from uniform distribution $U(0,10)$, which gives an average censoring proportion of $51.5 \%$.

Two marker interval sizes, 10 centiMorgans (cM) and $20 \mathrm{cM}$, were considered, and the Haldane's mapping function $\theta=\frac{1}{2}\left\{1-\exp \left(-2 d_{M}\right)\right\}$ was used to determine the relationship between recombination fraction $\theta$ and the genetic map distance $d_{M}$ (in Morgans). Two different positions of the QTL in the interval were studied: in the middle and at three quarters of the interval, respectively. We performed 500 runs of simulations with sample size of $n=200$. The number of iterations used in the EM algorithm was restricted to a maximum of 30 to save computation time in the simulation. From our experience, the algorithm usually converges within 30 iterations, but if convergence was not reached in 30 steps for some simulation runs, the values of the parameters at the 30 th step were used for parameter estimates. Simulation results under different interval sizes are summarized in Tables 1 and 2, respectively.

All the parameter estimates have negligible biases and the estimated standard errors (SEE) are all close to the sample standard errors (SSE) for both methods. Other baseline functions, for example, Weibull, were also adopted, and similar results were obtained (results not shown in the article). Variance estimates from Method 2 give slightly better coverage probabilities on $\left(\beta, \beta_{g}\right)$ than Method 1 (see $\beta$ rows in Tables 2 and 3). However, the EM-aided numerical differential method (Method 2) may occasionally encounter numerical difficulties. For example, Method 2 did not yield a positive definite variance-covariance matrix for a few (less than $5 \%$ ) of the simulation runs.

In our simulations, both methods underestimate the standard errors for $\beta$ 's slightly and thus the empirical coverage probabilities of $95 \%$ confidence intervals for $\beta$ 's are a little bit lower than the nominal level, especially in the simulations using Method 1. One possible reason may be that approximations are used for calculating the conditional expectations of 
Table 1

Simulation results for marker distance $=20 \mathrm{cM}(\theta=0.165)$

\begin{tabular}{|c|c|c|c|c|c|c|}
\hline Parameter & Bias & SSE & $\mathrm{SEE}^{1}$ & $\mathrm{CP}^{1}$ & $\mathrm{SEE}^{2}$ & $\mathrm{CP}^{2}$ \\
\hline & \multicolumn{6}{|c|}{$\mathrm{QTL}=10 \mathrm{cM}(r=0.091)$} \\
\hline$\gamma_{0}$ & -0.021 & 0.354 & 0.333 & 0.956 & 0.333 & 0.948 \\
\hline$\gamma_{1}$ & 0.105 & 0.405 & 0.388 & 0.944 & 0.388 & 0.942 \\
\hline$\gamma_{g}$ & -0.089 & 0.459 & 0.432 & 0.952 & 0.423 & 0.946 \\
\hline$\beta_{1}$ & -0.017 & 0.252 & 0.217 & 0.914 & 0.249 & 0.940 \\
\hline$\beta_{g}$ & 0.014 & 0.338 & 0.298 & 0.918 & 0.328 & 0.932 \\
\hline$r^{*}$ & 0.023 & 0.689 & 0.776 & 1.000 & 0.761 & 1.000 \\
\hline \multirow[t]{2}{*}{$r$} & 0.001 & 0.024 & 0.028 & 0.954 & 0.027 & 0.946 \\
\hline & \multicolumn{6}{|c|}{$\mathrm{QTL}=15 \mathrm{cM}(r=0.13)$} \\
\hline$\gamma_{0}$ & -0.006 & 0.362 & 0.333 & 0.950 & 0.332 & 0.950 \\
\hline$\gamma_{1}$ & 0.115 & 0.400 & 0.389 & 0.942 & 0.389 & 0.942 \\
\hline$\gamma_{g}$ & -0.129 & 0.466 & 0.430 & 0.948 & 0.419 & 0.944 \\
\hline$\beta_{1}$ & -0.021 & 0.257 & 0.217 & 0.908 & 0.243 & 0.928 \\
\hline$\beta_{g}$ & -0.001 & 0.337 & 0.294 & 0.910 & 0.316 & 0.932 \\
\hline$r^{*}$ & -0.048 & 0.828 & 0.962 & 0.966 & 0.934 & 0.966 \\
\hline$r$ & -0.001 & 0.022 & 0.024 & 0.946 & 0.023 & 0.940 \\
\hline
\end{tabular}

$\mathrm{SSE}=$ sample standard deviation; $\mathrm{SEE}^{k}=$ mean estimated standard error using method $k(k=1,2) ; \mathrm{CP}^{k}=$ the empirical coverage probability of a $95 \%$ Wald-type confidence interval using method $k(k=1,2)$.

Standard error for $r$ is approximated by $\delta$-method from standard error of $r^{*}$.

Table 2

Simulation results for marker distance $=10 c M(\theta=0.091)$

\begin{tabular}{lrlllll}
\hline \hline Parameter & Bias & SSE & SEE $^{1}$ & $\mathrm{CP}^{1}$ & $\mathrm{SEE}^{2}$ & $\mathrm{CP}^{2}$ \\
\hline & & $\mathrm{QTL}$ & $=5 \mathrm{cM}$ & $(r=0.0476)$ \\
$\gamma_{0}$ & -0.021 & 0.335 & 0.322 & 0.950 & 0.323 & 0.950 \\
$\gamma_{1}$ & 0.100 & 0.385 & 0.381 & 0.964 & 0.382 & 0.964 \\
$\gamma_{g}$ & -0.102 & 0.427 & 0.400 & 0.946 & 0.398 & 0.948 \\
$\beta_{1}$ & -0.026 & 0.255 & 0.216 & 0.922 & 0.243 & 0.938 \\
$\beta_{2}$ & 0.005 & 0.315 & 0.274 & 0.918 & 0.308 & 0.940 \\
$r^{*}$ & 0.005 & 0.938 & 1.097 & 1.000 & 1.081 & 1.000 \\
$r$ & 0.000 & 0.017 & 0.020 & 0.934 & 0.020 & 0.928 \\
& & $\mathrm{QTL}=7.5 \mathrm{cM}$ & $(r=0.0696)$ & \\
$\gamma_{0}$ & -0.021 & 0.341 & 0.322 & 0.936 & 0.322 & 0.936 \\
$\gamma_{1}$ & 0.107 & 0.390 & 0.381 & 0.944 & 0.381 & 0.944 \\
$\gamma_{g}$ & -0.112 & 0.427 & 0.398 & 0.946 & 0.395 & 0.944 \\
$\beta_{1}$ & -0.020 & 0.251 & 0.217 & 0.928 & 0.239 & 0.934 \\
$\beta_{2}$ & 0.001 & 0.321 & 0.273 & 0.908 & 0.301 & 0.940 \\
$r^{*}$ & -0.008 & 1.024 & 1.307 & 0.990 & 1.307 & 0.990 \\
$r$ & -0.000 & 0.015 & 0.018 & 0.930 & 0.018 & 0.926 \\
\hline
\end{tabular}

See the footnote of Table 1 .

$$
\frac{\sum_{j \in R_{i}} \eta_{j} e^{\beta Z_{j}+\beta_{g} G_{j}} Z_{j}}{\sum_{j \in R_{i}} \eta_{j} e^{\beta Z_{j}+\beta_{g} G_{j}}} \text { and } \frac{\sum_{j \in R_{i}} \eta_{j} e^{\beta Z_{j}+\beta_{g} G_{j}} G_{j}}{\sum_{j \in R_{i}} \eta_{j} e^{\beta Z_{j}+\beta_{g} G_{j}}}
$$

which are needed in calculation of variance of Method 1. Direct calculation of the conditional expectations of these two ratios are very complicated, and thus we propose to approximate them by

$$
\begin{aligned}
& \frac{\sum_{j \in R_{i}} E\left(\eta_{j} e^{\beta Z_{j}+\beta_{g} G_{j}} Z_{j} \mid \mathcal{O}, \hat{\alpha}, \hat{\Lambda}\right)}{\sum_{j \in R_{i}} E\left(\eta_{j} e^{\beta Z_{j}+\beta_{g} G_{j}} \mid \mathcal{O}, \hat{\alpha}, \hat{\Lambda}\right)} \text { and } \\
& \frac{\sum_{j \in R_{i}} E\left(\eta_{j} e^{\beta Z_{j}+\beta_{g} G_{j}} G_{j} \mid \mathcal{O}, \hat{\alpha}, \hat{\Lambda}\right)}{\sum_{j \in R_{i}} E\left(\eta_{j} e^{\beta Z_{j}+\beta_{g} G_{j}} \mid \mathcal{O}, \hat{\alpha}, \hat{\Lambda}\right)} .
\end{aligned}
$$

In Li and Thompson (1997), multiple imputation techniques were used in both EM algorithm and variance estimation so that no such approximations were needed. However, the computation using imputations is quite demanding for our situation. The proposed approximations directly use the results from the EM algorithm and approach the true conditional expectations when sample size increases.

For estimating the position of the QTL, Kao and Zeng (1997) noted that accuracy increases when the trait variation controlled by QTL (heritability) enlarges. However, it is more difficult to explicitly control the heritability (variance) for the survival models, where traditional focus is on hazards and risk. In our simulations, the estimation of $r$ is virtually unbiased, but the coverage probability often exceeds the nominal level, which may indicate insufficiency of information contained in the data to locate $r$ accurately. Thus a good experimental design is very important to obtain a precise confidence interval for the QTL location. Furthermore, fine mapping steps are often necessary in the process of QTL analysis, for example, association study using single nucleotide polymorphisms (SNP).

\subsection{A Real Data Example}

To illustrate the proposed proportional hazards cure model on interval mapping of QTL, we analyzed a real data set that has been investigated by Boyartchuk et al. (2001) and Broman (2003). The data consist of survival times of 116 female mice after infection with Listeria monocytogenes from an intercross between the BALB/cByJ and C57BL/6ByJ strains. The target interval of our interest is located on chromosome 13 between the markers D13M99 and D13M147. Table 3 summarizes our estimates of the QTLs' long-term effects on susceptibility and short-term effects on survival times for susceptible mice and the position of the QTL inside the target interval.

The recombination fraction between the two markers is 0.078 estimated by R/qtl (Broman et al., 2003). The variance estimates are computed using both methods discussed in the preceeding section. The estimated QTL location $\hat{r}=0.068$ suggests closeness to the right-side marker D13M147, but great confidence cannot be placed in this conclusion because the interval for the location parameter is relatively wide. In Table 3, the results also indicate that the susceptibility of the $Q Q$ group is significantly higher than the other two groups ( $p$ value $<0.05)$. However, there is not enough evidence to detect the difference between the $Q q$ and $q q$ groups, although the $Q q$ group tends to have more late events. Similar results were also found in Broman (2003). 
Table 3

Estimates of parameters and standard errors

\begin{tabular}{lcccc}
\hline \hline & & Long-term effects & Short-term effects & Position \\
\hline Parameters & $\gamma_{0}$ & $\gamma_{g 1}(\mathrm{QQ}) \gamma_{g 2}(\mathrm{Qq})$ & $\beta_{g 1}(\mathrm{QQ}) \beta_{g 2}(\mathrm{Qq})$ & $r^{*}(r)$ \\
Estimate & 0.446 & 1.9151 .123 & $0.673-0.918$ & $1.942(0.068)$ \\
SD1 & 0.520 & 0.7600 .889 & 0.3500 .373 & 1.790 \\
SD2 & 0.543 & 0.7741 .231 & 0.4380 .521 & 1.829 \\
\hline
\end{tabular}

SD1 $=$ standard error estimates obtained by applying Method 1; SD2 $=$ standard error estimates obtained by applying Method 2.

In Figure 1b, we plotted the predicted survival curves using the proportional hazards mixture cure model for the three groups of mice with respect to the QTL genotypes $Q Q, Q q$, and $q q$, respectively. The curves look very similar to the observed Kaplan-Meier curves plotted in Figure 1a. To illustrate the sole QTL's short-term effects on survival times of susceptible mice, we plotted in Figure 2 the predicted survival distribution for susceptible mice with QTL genotypes $Q Q, Q q$, and $q q$, respectively. From Figure 1, it can be seen that those mice with genotype $q q$ have a much higher chance (around $40 \%$ ) to be fully recovered from the infection compared to the $Q Q$ group (around 10\%). But the susceptible mice of these two groups behave similarly and both experience the events fairly early (Figure 2). In addition, susceptible mice with the heterozygous genotype $Q q$ at the QTL tend to have more late event times than those with homozygous genotypes $Q Q$ or $q q$ at the QTL (Figure 2).

\section{Extension to Multiple Intervals}

In some situations, the uncertainty of the target chromosomal region may extend over multiple marker intervals. The choice of chromosomal region may depend on many factors, for example, the shape of the log of odds (LOD) score, the distribution of the markers, and the availability and strength of other scientific evidence, etc. To address this important issue, we propose an extended estimation procedure that works for multiple marker intervals. Consider $J$ intervals of inter-

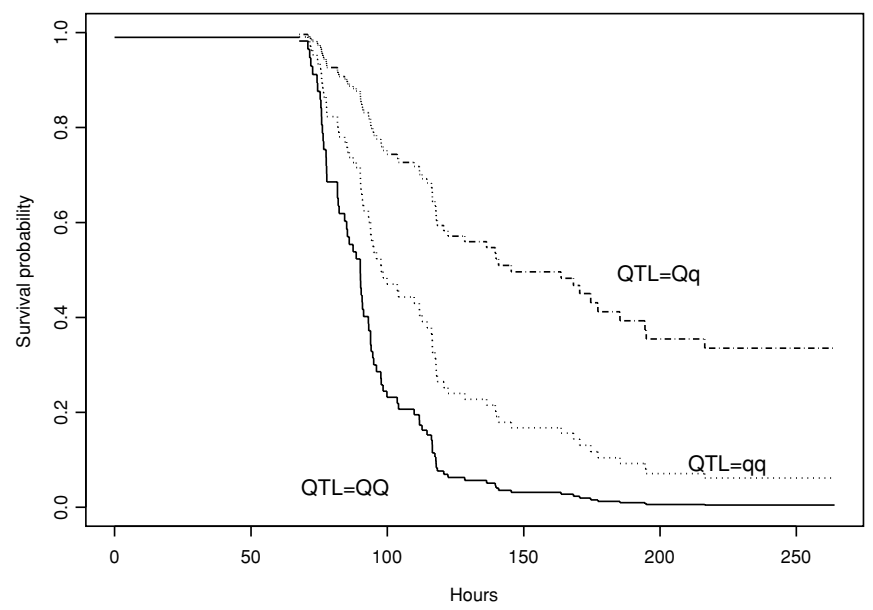

Figure 2. Predicted survival distribution for susceptible subjects. est, defined by adjacent markers $M^{0}, M^{1}, \ldots, M^{J}$. In the $j$ th interval, the EM algorithm described in Section 3 is applied to maximize the likelihood function confined in this interval and thereby yields the MLE $\left(\hat{\boldsymbol{\alpha}}^{j}, \hat{\Lambda}^{j}\right)$. Then at each estimate, we evaluate the observed-data likelihood function, which is easily derived from (4). Specifically, for backcross design, the likelihood function can be written as

$$
\begin{aligned}
L_{O}(\boldsymbol{\alpha}, \Lambda ; j)=\prod_{i=1}^{n}\left[p_{i}(j)\{(\right. & \left.\left(Y_{i}\right) e^{\beta^{\prime} Z_{i}+\beta_{g}}\right)^{\delta_{i}} e^{-\Lambda\left(Y_{i} ; \theta\right) \exp \left(\beta^{\prime} Z_{i}+\beta_{g}\right)} \\
\times & \left.\frac{e^{\gamma^{\prime} Z_{i}^{*}+\gamma_{g}}}{1+e^{\gamma^{\prime} Z_{i}^{*}+\gamma_{g}}}+\frac{1-\delta_{i}}{1+e^{\gamma^{\prime} Z_{i}^{*}+\gamma_{g}}}\right\} \\
+\left(1-p_{i}(j)\right) & \left\{\left(\lambda\left(Y_{i}\right) e^{\beta^{\prime} Z_{i}}\right)^{\delta_{i}} e^{-\Lambda\left(Y_{i} ; \theta\right) \exp \left(\beta^{\prime} Z_{i}\right)}\right. \\
& \left.\left.\times \frac{e^{\gamma^{\prime} Z_{i}^{*}}}{1+e^{\gamma^{\prime} Z_{i}^{*}}}+\frac{1-\delta_{i}}{1+e^{\gamma^{\prime} Z_{i}^{*}}}\right\}\right], \quad(8)
\end{aligned}
$$

where $p_{i}(j)=\operatorname{Pr}\left(G_{i}=1 \mid M_{i}^{j-1}, M_{i}^{j}\right)$. We use $\hat{L}_{O}(j)$ to denote the value of $L_{O}$ at $\left(\hat{\boldsymbol{\alpha}}^{j}, \hat{\Lambda}^{j}\right)$ and define the value of the MLE on these $J$ intervals as $(\hat{\boldsymbol{\alpha}}, \hat{\Lambda})=\left(\hat{\boldsymbol{\alpha}}^{j *}, \hat{\Lambda}^{j *}\right)$, where $j^{*}=\operatorname{argmax}_{1 \leq j \leq J} \hat{L}_{O}(j)$. To obtain the variance estimates of $(\hat{\boldsymbol{\alpha}}, \hat{\Lambda})$ and the corresponding confidence intervals, we employ the bootstrap method by resampling the subjects with replacement (Efron, 1979). Let $\left\{\left(\hat{\boldsymbol{\alpha}}_{b}, \hat{\Lambda}_{b}\right), b=1, \ldots, B\right\}$ denote the bootstrapped estimates, where $B$ is the number of bootstrap replications. Then the confidence intervals for the parameter estimates $\hat{\boldsymbol{\alpha}}$ can be constructed using the Wald-type method with the standard errors of $\left\{\hat{\boldsymbol{\alpha}}_{b}\right\}$ obtained from the bootstrap.

Additional simulations were conducted to assess the performance of the proposed estimation method over multiple intervals. Here we choose to present one of the simulation results because others are similar. In this simulation study, we considered a chromosomal region of $30 \mathrm{cM}$ with four equally spaced markers. The true QTL location $d$ was set at the middle of the second interval $\left(d_{0}=15 \mathrm{cM}\right)$. The setup of the other parameter values remained the same as in Section 4.1, more specifically, $\left\{\gamma_{0}, \gamma_{1}, \gamma_{g}, \beta_{1}, \beta_{g}\right\}=\{1,0.5,-2,0.5,-1\}$. Each run of the simulation was conducted with a sample size of $n=200$ and with $B=500$ bootstrap replications. We performed 100 runs of simulations. The results are summarized in Table 4.

From the results in Table 4, it is clear that all the point estimates have reasonably small biases and the standard error estimates obtained using the bootstrap method provide good 
Table 4

Simulation results for multiple intervals

\begin{tabular}{lrccc}
\hline \hline Parameter & \multicolumn{1}{c}{ Bias } & SSE & SEE & CP \\
\hline$\gamma_{0}$ & -0.031 & 0.327 & 0.370 & 0.96 \\
$\gamma_{1}$ & 0.154 & 0.386 & 0.427 & 0.96 \\
$\gamma_{g}$ & -0.109 & 0.446 & 0.465 & 0.97 \\
$\beta_{1}$ & 0.010 & 0.239 & 0.267 & 0.97 \\
$\beta_{g}$ & 0.046 & 0.328 & 0.365 & 0.96 \\
$d$ & 0.263 & 3.623 & 4.095 & 0.95 \\
\hline
\end{tabular}

$\mathrm{SSE}=$ sample standard deviation; $\mathrm{SEE}=$ mean estimated standard error using bootstrap method; $\mathrm{CP}=$ the empirical coverage probability of a $95 \%$ Wald-type confidence interval.

approximation to the sampling standard error, especially on the parameters $\left(\beta_{1}, \beta_{g}\right)$ and $d$. The coverage probabilities are close to the nominal level. Moreover, in our simulations, the confidence intervals for the QTL location parameter were not always confined within a single interval. In our simulations, although there were $18 \%$ of the QTL location parameter estimates that fell outside the correct interval, the proposed multiple-interval estimation procedure still yields a confidence interval with satisfactory coverage probability for the QTL location parameter. It is clear that a single-interval-based estimation approach would not perform well in this specific setup. Thus it is important not to choose a candidate chromosomal region that is too small so that the true QTL is likely to fall out of the region.

\section{Discussion}

In this article, we proposed the proportional hazards mixture cure model for joint estimation of QTL position, QTL effects, and environmental effects using time-to-event traits. The proposed model is semiparametric in nature and can naturally handle the situation that the underlying population is a mixture of susceptible and nonsusceptible subjects. The candidate chromosomal region for QTL estimation can either be a single marker interval or a region extending over multiple intervals. This method is particularly valuable for studying candidate intervals where the QTL position has been implicated in previous linkage studies.

As pointed out by an associate editor, using the same data set that has been used for genome-wise QTL detection may incur serious selection bias. The selection bias issue has been discussed in the literature (e.g., Utz, Melchinger, and Schön, 2000; Göring, Terwilliger, and Blangero, 2001). In particular, genetic data from independent replication studies can be used to avoid serious selection bias in QTL estimation.

The statistical method developed in this article for QTL mapping can be applied to handle the proportional hazards cure model with general missing covariates or frailty for clustered data. As we discussed before, the missing covariates in the model considered for QTL mapping are all discrete. When some of the missing covariates are continuous, numerical integration might be employed for computing the conditional expectations used in the EM algorithm.

\section{ACKNOWLEDGEMENTS}

The authors would like to thank Professor Karl Broman for kindly providing the Listeria data and $\mathrm{R} / \mathrm{qtl}$ package. We are grateful to the editor, an associate editor, and a referee for their valuable suggestions and constructive comments. We also appreciate the careful reading by Linda Rolnitzky. The work of the first and the third authors was supported in part by NIEHS grant ES-000260 and NCI grant CA-016087. The work of the second author was supported in part by NSF grant DMS-0504269.

\section{REFERENCES}

Boyartchuk, V. L., Broman, K. W., Mosher, R. E., D’Orazio, S. E. F., Starnbach, M. N., and Dietrich, W. F. (2001). Multigenic control of Listeria monocytogenes susceptibility in mice. Nature Genetics 27, 259-260.

Breslow, N. E. (1975). Analysis of survival data under the proportional hazards model. International Statistical Review 43, $45-58$.

Broman, K. W. (2003). Mapping quantitative trait loci in the case of a spike in the phenotype distribution. Genetics 163, 1169-1175.

Broman, K. W., Wu, H., Sen, S., and Churchill, G. A. (2003). R/qtl: QTL mapping in experimental crosses. Bioinformatics 19, 889-890.

Chen, H. Y. and Little, R. J. A. (1999). Proportional hazards regression with missing covariates. Journal of the American Statistical Association 94, 896-908.

Cox, D. R. (1972). Regression models and life tables (with discussion). Journal of the Royal Statistical Society, Series B 34, 187-220.

Darvasi, A., Weinreb, A., Minke, V., Weller, J. I., and Soller, M. (1993). Detecting marker-QTL linkage and estimating QTL gene effect and map location using a saturated genetic map. Genetics 134, 943-951.

Dempster, A. P., Laird, N. M., and Rubin, D. B. (1977). Maximum likelihood from incomplete data via the EM algorithm. Journal of the Royal Statistical Society, Series B 39, 1-38.

Doerge, R. W., Zeng, Z.-B., and Weir, B. S. (1997). Statistical issues in the search for genes affecting quantitative traits in experimental populations. Statistical Science 12, 195219.

Efron, B. (1979). Bootstrap methods: Another look at the jackknife. Annals of Statistics 7, 1-26.

Feenstra, B. and Skovgarrd, I. M. (2004). A quantitative trait locus mixture model that avoids spurious LOD score peaks. Genetics 167, 959-965.

Göring, H. H., Terwilliger, J. D., and Blangero, J. (2001). Large upward bias in estimation of locus-specific effects from genomewide scans. American Journal of Human Genetics 69, 1357-1369.

Johansen, S. (1983). An extension of Cox's regression model. International Statistical Review 51, 165-174.

Kalbfleisch, J. D. and Prentice, R. L. (2002). The Statistical Analysis of Failure Time Data, 2nd edition. Hoboken, New Jersey: Wiley.

Kao, C. H. and Zeng, Z.-B. (1997). General formulas for obtaining the MLEs and the asymptotic variancecovariance matrix in mapping quantitative trait loci when using the EM algorithm. Biometrics 53, 653-665.

Klein, J. P. (1992). Semiparametric estimation of random effects using the Cox model based on the EM algorithm. Biometrics 48, 785-806. 
Kruglyak, L. and Lander, E. S. (1995). A non-parametric approach for mapping quantitative trait loci. Genetics $\mathbf{1 3 9}$, 1421-1428.

Kuk, A. Y. C. and Chen, C.-H. (1992). A mixture model combining logistic regression with proportional hazards regression. Biometrika 79, 531-541.

Lander, E. S. and Botstein, D. (1989). Mapping Mendelian factors underlying quantitative traits using RFLP linkage maps. Genetics 121, 185-199.

Lander, E. S. and Schork, N. J. (1994). Genetic dissection of complex traits. Science 265, 2037-2048.

Li, H. and Thompson, E. (1997). Semiparametric estimation of major gene and family-specific random effects for age of onset. Biometrics 53, 282-293.

Louis, T. A. (1982). Finding the observed information matrix when using the EM algorithm. Journal of the Royal Statistical Society, Series B 44, 226-233.

Lu, W. and Ying, Z. (2004). On semiparametric transformation cure models. Biometrika 91, 331-343.

Meilijson, E. (1989). A fast improvement to the EM algorithm on its own terms. Journal of the Royal Statistical Society, Series B 51, 127-138.

Murphy, S. A. and Van der Vaart, A. W. (2000). On profile likelihood (with discussion). Journal of the American Statistical Association 95, 449-485.

Peng, Y. and Dear, K. B. G. (2000). A nonparametric mixture model for cure rate estimation. Biometrics 56, 237-243.

Shalom, A. and Darvasi, A. (2002). Experimental designs for QTL fine mapping in rodents. In Quantitative Trait Loci:
Methods and Protocols, N. J. Camp and A. Cox (eds). Totowa, New Jersey: Humana Press.

Sy, J. P. and Taylor, J. M. G. (2000). Estimation in a Cox proportional hazards cure model. Biometrics 56, 227236.

Utz, H. F., Melchinger, A. E., and Schön, C. C. (2000). Bias and sampling error of the estimated proportion of genotypic variance explained by quantitative trait loci determined from experimental data in maize using cross validation and validation with independent samples. $G e-$ netics 154, 1839-1849.

Received February 2005. Revised February 2006. Accepted March 2006.

\section{APPENDIX}

In the $k$ th step of the EM algorithm, conditional expectation of complete-data log likelihood, $\tilde{l}_{C}\left(\boldsymbol{\alpha}, \Lambda_{0} \mid \boldsymbol{\alpha}^{(k)}, \Lambda_{0}^{(k)}\right)$, is

$$
\begin{aligned}
\sum_{i=1}^{n}[ & \gamma^{\prime} Z_{i}^{*} P_{\eta_{i}}^{(k)}+\gamma_{g} P_{\eta_{i} G_{i}}^{(k)}-\log \left(1+e^{\gamma^{\prime} Z_{i}^{*}+\gamma_{g}}\right) P_{G_{i}}^{(k)} \\
& -\log \left(1+e^{\gamma^{\prime} Z_{i}^{*}}\right)\left(1-P_{G_{i}}^{(k)}\right) \\
& +\delta_{i}\left\{\log \left(\lambda_{0}\left(Y_{i}\right)\right)+\beta^{\prime} Z_{i}+\beta P_{G_{i}}^{(k)}\right\} \\
& -\Lambda_{0}\left(Y_{i}\right) e^{\beta^{\prime} Z_{i}+\beta_{g}} P_{\eta_{i} G_{i}}^{(k)}-\Lambda_{0}\left(Y_{i}\right) e^{\beta^{\prime} Z_{i}}\left(P_{\eta_{i}}^{(k)}-P_{\eta_{i} G_{i}}^{(k)}\right) \\
& \left.+P_{G_{i}}^{(k)} \log \left(p_{i}\right)+\left(1-P_{G_{i}}^{(k)}\right) \log \left(1-p_{i}\right)\right] .
\end{aligned}
$$

